\title{
BMJ Open Evidence-informed recommendations for rehabilitation with older adults living with HIV: a knowledge synthesis
}

\author{
Kelly K O'Brien, ${ }^{1,2,3}$ Patricia Solomon, ${ }^{2}$ Barry Trentham, ${ }^{4}$ Duncan MacLachlan, ${ }^{5}$ \\ Joy MacDermid, ${ }^{2}$ Anne-Marie Tynan, ${ }^{6}$ Larry Baxter, ${ }^{3}$ Alan Casey, ${ }^{7}$ \\ William Chegwidden, ${ }^{8}$ Greg Robinson, ${ }^{3}$ Todd Tran, ${ }^{9}$ Janet Wu, ${ }^{6}$ Elisse Zack ${ }^{3}$
}

To cite: O'Brien KK, Solomon P, Trentham B, et al. Evidence-informed recommendations for rehabilitation with older adults living with HIV: a knowledge synthesis. BMJ Open 2014;4:e004692. doi:10.1136/bmjopen-2013004692

- Prepublication history and additional material is available. To view please visit the journal (http://dx.doi.org/ 10.1136/bmjopen-2013004692).

Received 15 December 2013 Revised 7 April 2014 Accepted 10 April 2014

CrossMark

For numbered affiliations see end of article.

Correspondence to Dr Kelly K O'Brien; kelly.obrien@utoronto.ca

\section{ABSTRACT}

Objective: Our aim was to develop evidence-informed recommendations for rehabilitation with older adults living with HIV.

Design: We conducted a knowledge synthesis, combining research evidence specific to HIV, rehabilitation and ageing, with evidence on rehabilitation interventions for common comorbidities experienced by older adults with HIV.

Methods: We included highly relevant HIV-specific research addressing rehabilitation and ageing (stream $A$ ) and high-quality evidence on the effectiveness of rehabilitation interventions for common comorbidities experienced by older adults ageing with HIV (stream B). We extracted and synthesised relevant data from the evidence to draft evidence-informed recommendations for rehabilitation. Draft recommendations were refined based on people living with HIV (PLHIV) and clinician experience, values and preferences, reviewed by an interprofessional team for Grading of Recommendations Assessment, Development, and Evaluation (GRADE) (quality) rating and revision and then circulated to PLHIV and clinicians for external endorsement and final refinement. We then devised overarching recommendations to broadly guide rehabilitation with older adults living with HIV.

Results: This synthesis yielded 8 overarching and 52 specific recommendations. Thirty-six specific recommendations were derived from 108 moderate-level or high-level research articles (meta-analyses and systematic reviews) that described the effectiveness of rehabilitation interventions for comorbidities that may be experienced by older adults with HIV. Recommendations addressed rehabilitation interventions across eight health conditions: bone and joint disorders, cancer, stroke, cardiovascular disease, mental health challenges, cognitive impairments, chronic obstructive pulmonary disease and diabetes. Sixteen specific recommendations were derived from 42 research articles specific to rehabilitation with older adults with HIV. The quality of evidence from which these recommendations were derived was either low or very low, consisting primarily of narrative reviews or descriptive studies with small sample sizes. Recommendations addressed approaches to rehabilitation assessment and interventions, and contextual factors to consider for rehabilitation with older adults living with HIV.

\section{Strengths and limitations of this study}

- We developed evidence-informed recommendations for rehabilitation with older adults living with HIV using a complex knowledge synthesis of two distinct areas of literature while incorporating PLHIV and clinician preferences throughout.

- Fifty-two recommendations were developed.

- Thirty-six specific recommendations were derived from 108 moderate-level or high-level research articles that described the effectiveness of rehabilitation interventions for comorbidities that may be experienced by older adults with HIV. Recommendations addressed rehabilitation interventions across eight health conditions commonly experienced by older adults with HIV.

- Sixteen specific recommendations were derived from 42 research articles specific to rehabilitation of older adults with HIV.

- To our knowledge, these are the first evidence-informed recommendations for rehabilitation developed specifically for older adults living with HIV.

- Recommendations address approaches to rehabilitation assessment and interventions, and contextual factors to consider for rehabilitation with older adults living with HIV.

Conclusions: These evidence-informed recommendations provide a guide for rehabilitation with older adults living with HIV.

\section{INTRODUCTION}

As adults age with HIV, more individuals are living with the physical, social and psychological consequences of the disease, long-term treatment and comorbidities associated with ageing. ${ }^{1-4}$ For many, HIV is experienced as a chronic illness whereby individuals experience a range of health-related challenges known as disability, including symptoms and impairments 
(eg, fatigue, weakness, pain), difficulties with day-to-day activities (eg, household chores), challenges to social inclusion (eg, ability to work) and uncertainty or worrying about future health as they age..$^{5-7}$ Premature onset of cardiovascular disease $(\mathrm{CVD}),{ }^{8}$ diabetes, ${ }^{8}$ bone and joint disorders, ${ }^{9}$ neurocognitive disorders ${ }^{10}$ and non-AIDS-defining cancers ${ }^{11}$ further add to the complexity of disability experienced by adults ageing with HIV. ${ }^{12-16}$ Rehabilitation has become an increasingly important strategy to address disability experienced by adults ageing with HIV and specifically older adults living with comorbidities. ${ }^{17}$

Rehabilitation is broadly defined as any service or health provider that may address or prevent impairments, activity limitations or social participation restrictions experienced by an individual. ${ }^{17}$ Rehabilitation assists in managing the health-related challenges or disability associated with HIV such as adverse effects of medications, fatigue, pain, neuropathy, mental health problems, cognitive problems and issues related to income and vocational support. Rehabilitation approaches such as physical therapy and occupational therapy are well established in complex chronic disease management and are associated with improvements in health outcomes in $\mathrm{CVD},{ }^{18}$ stroke $^{19}$ and cancer. ${ }^{20}$ However, rehabilitation in the context of HIV is still emerging. Few rehabilitation professionals work with people living with HIV (PLHIV) highlighting a gap in service provision and need for further HIV knowledge, training and clinical guidance. ${ }^{21}$ Evidence-informed guidelines are essential to enhance awareness among clinicians, researchers, educators and PLHIV and to optimise rehabilitation with older adults with HIV.

No known guidelines specific to HIV rehabilitation and ageing exist. Developing evidence-informed recommendations in an emerging area of practice is challenging when high levels of evidence in the form of systematic reviews and meta-analyses are often not available. Such is the case with HIV, which has transitioned from an acute fatal illness to a chronic condition since the advent of combination antiretroviral therapies that became available in developed countries in the mid-1990s. Combining lower level evidence on emerging issues of HIV and ageing with higher level evidence on rehabilitation interventions for other health conditions experienced by older adults with HIV can provide a strong foundation for the development of evidence-informed recommendations. Our aim was to develop evidence-informed recommendations for rehabilitation with older adults living with HIV.

\section{METHODS}

We conducted a knowledge synthesis combining two streams of evidence: (A) highly relevant HIV-specific evidence addressing rehabilitation and ageing and (B) high-quality evidence on the effectiveness of non-pharmacological rehabilitation interventions for comorbidities commonly experienced by older adults ageing with HIV. Synthesising this evidence allowed us to consider emerging literature specific to HIV and ageing while taking advantage of existing high-level evidence on interventions for common conditions experienced by older adults and customising it to older adults with HIV.

This research was led by an interdisciplinary team of researchers, educators, health providers with expertise in HIV, ageing and rehabilitation and PLHIV with lived experience ageing with HIV. The team engaged in all aspects of this study including the identification, appraisal and synthesis of the literature, and development and refinement of the evidence-informed recommendations. We incorporated values and preferences of PLHIV and clinical expertise throughout. ${ }^{22}$

\section{Searching and identifying the literature and data extraction}

Stream A: Evidence specific to HIV, ageing and rehabilitation

We conducted a synthesis of published literature specific to HIV, ageing and rehabilitation. We searched electronic databases including MEDLINE, CINAHL, EMBASE, PsycINFO from 1980 to December 2010. Search terms included: HIV, ageing and rehabilitation, and were altered depending on the database. We included studies that addressed issues related to HIV, ageing (older adults: 50 years and older) and rehabilitation, and were published in the English language. We defined 'rehabilitation' as any non-pharmacological services, interventions or providers who address or prevent impairments, activity limitations and social participation restrictions experienced by an individual. ${ }^{17}$ Given this is an emerging area of literature, all study designs, including narrative reviews, were included.

\section{Stream B: High-level evidence on rehabilitation interventions specific to comorbidities that may be experienced by older} adults living with HIV

We searched for high-quality evidence (systematic reviews and meta-analyses) on the effectiveness of nonpharmacological rehabilitation interventions for comorbidities that may be experienced by older adults ageing with HIV. We searched electronic databases including MEDLINE, CINAHL, EMBASE, PsycINFO, the Cochrane Database of Systematic Reviews and the National Guideline Clearinghouse from 1980 to August 2011 for systematic reviews and meta-analyses related to common comorbidities. We included systematic reviews or meta-analyses that addressed one or more comorbidities experienced by adults living with HIV which included but were not limited to: bone and joint disorders, cancer, CVD, mental health, neurocognitive decline, cardiopulmonary disease, diabetes, and were published in English.

Five individuals independently reviewed abstracts from stream A and B evidence to determine their eligibility for inclusion. Where disagreements occurred, the full text was retrieved and a third reviewer determined final 
inclusion. ${ }^{23}$ Two individuals independently reviewed full articles for inclusion. In situations of disagreement reviewers discussed articles to reach consensus on final inclusion. Five individuals independently extracted data from the final group of included evidence onto a data charting form. Data extracted from stream A evidence included author, year, study location, study purpose, study design, intervention type and comparison group (if any), details of the intervention (if applicable), study population, sample size, outcome measures, key results, and authors' overall conclusions. For each article, reviewers documented their interpretations of important considerations and recommendations for HIV rehabilitation and ageing. Data extracted from stream B evidence included author, year, study purpose, study design (systematic review or meta-analysis), characteristics of participants, number of included studies, sample size, intervention(s) and comparison group (if any), frequency, intensity, time and type of each intervention, outcome measures, key results, and overall author conclusions. For each article, reviewers documented their interpretations of considerations for developing evidence informed recommendations with older adults living with HIV.

\section{Development of the recommendations}

We developed the evidence-informed recommendations using a three-phase iterative process involving (1) classification, assessing methodological quality, synthesis of the evidence and drafting the preliminary recommendations; (2) interprofessional team review, GRADING and revision of recommendations incorporating values and preferences; and (3) external endorsement and final refinement.

Phase 1: Classification, assessing methodological quality, synthesis of the evidence and drafting the preliminary recommendations

Our search yielded a total of 6664 independent citations (2512 from stream A and 4152 from stream B), of which 165 studies (50 studies from stream A and 115 studies from stream B) met our inclusion criteria. Overall, our phase 1 synthesis yielded 25 recommendations from stream A evidence and 49 recommendations from stream B evidence for a total of 74 preliminary recommendations (figure 1).

\section{Stream A: Evidence specific to HIV, rehabilitation and ageing} We classified the evidence ( $\mathrm{n}=50$ studies) based on 11 concepts to draft the preliminary recommendations ranging from overarching principles for rehabilitation with older adults living with HIV to interventions (figure 1). We then assessed the methodological quality of each included article and the quality of the collective group of evidence from each of the 11 key concepts used to draft each recommendation using GRADE methodological quality criteria. ${ }^{24-28}$ Two authors knowledgeable in HIV, ageing and rehabilitation (KKOB, AMT) independently synthesised the extracted data using directed content analysis techniques ${ }^{29}$ and formulated key themes surrounding rehabilitation assessment and treatment that informed the recommendations. One author (KKOB) then drafted 25 preliminary recommendations by synthesising results and conclusions from each collective group of evidence. Subsequently, two authors (KKOB, PS) met to review the accuracy of the content analysis and collectively agreed on the 74 preliminary evidence-informed recommendations specific to HIV, ageing and rehabilitation.

\section{Stream B: High-level evidence on rehabilitation interventions for common comorbidities}

We grouped stream B evidence based on comorbidities experienced by older adults living with HIV, followed by the respective intervention. We classified the evidence based on 11 areas (bone and joint disorders, cancer, stroke, CVD, mental health challenges, cognitive impairments, Parkinson's disease, chronic obstructive pulmonary disease (COPD), diabetes, older adults, HIV; figure 1). We assessed the methodological quality of each article and the quality of evidence from each collective area of focus used to draft each recommendation using the GRADE criteria. ${ }^{24-28}$ Two authors (KKOB, AMT) independently synthesised the recommendations from the meta-analyses and systematic reviews using directed content analysis techniques $^{29}$ surrounding assessment, treatment intervention, intensity, progression of intensity and health outcomes for each comorbidity. One author (KKOB) then drafted a total of 49 preliminary recommendations from the 115 included articles by synthesising each collective group of study results and overall conclusions. Two authors (KKOB, PS) met to review the accuracy of the synthesis to collectively determine preliminary evidence-informed recommendations for each of the comorbidities. The resulting 49 recommendations for stream B spanned 11 areas of focus: bone and joint disorders (6 recommendations), cancer (8), stroke (12), CVD (7), mental health challenges (4), cognitive impairments (3), Parkinson's disease (1), COPD (4), diabetes (1), older adults (2) and HIV (1).

Phase 2: Research team GRADING of recommendations and incorporating values and preferences among PLHIV and clinicians

We circulated the 74 preliminary recommendations to researchers, PLHIV and clinicians on the synthesis team in order to obtain GRADE ratings of the recommendations and incorporate individual experiences, values and preferences. For each recommendation, the team member indicated the GRADE rating incorporating quality of the evidence and the extent to which the recommendation was applicable to older adults living with HIV. GRADE rating at this stage included four levels ${ }^{24-28}$ : High - fully endorse or strongly recommend. This recommendation would be appropriate for the majority of older adults living with HIV; suggested wording of the recommendation would include, "we should or should not do'; Moderate—moderately endorse or recommend. 


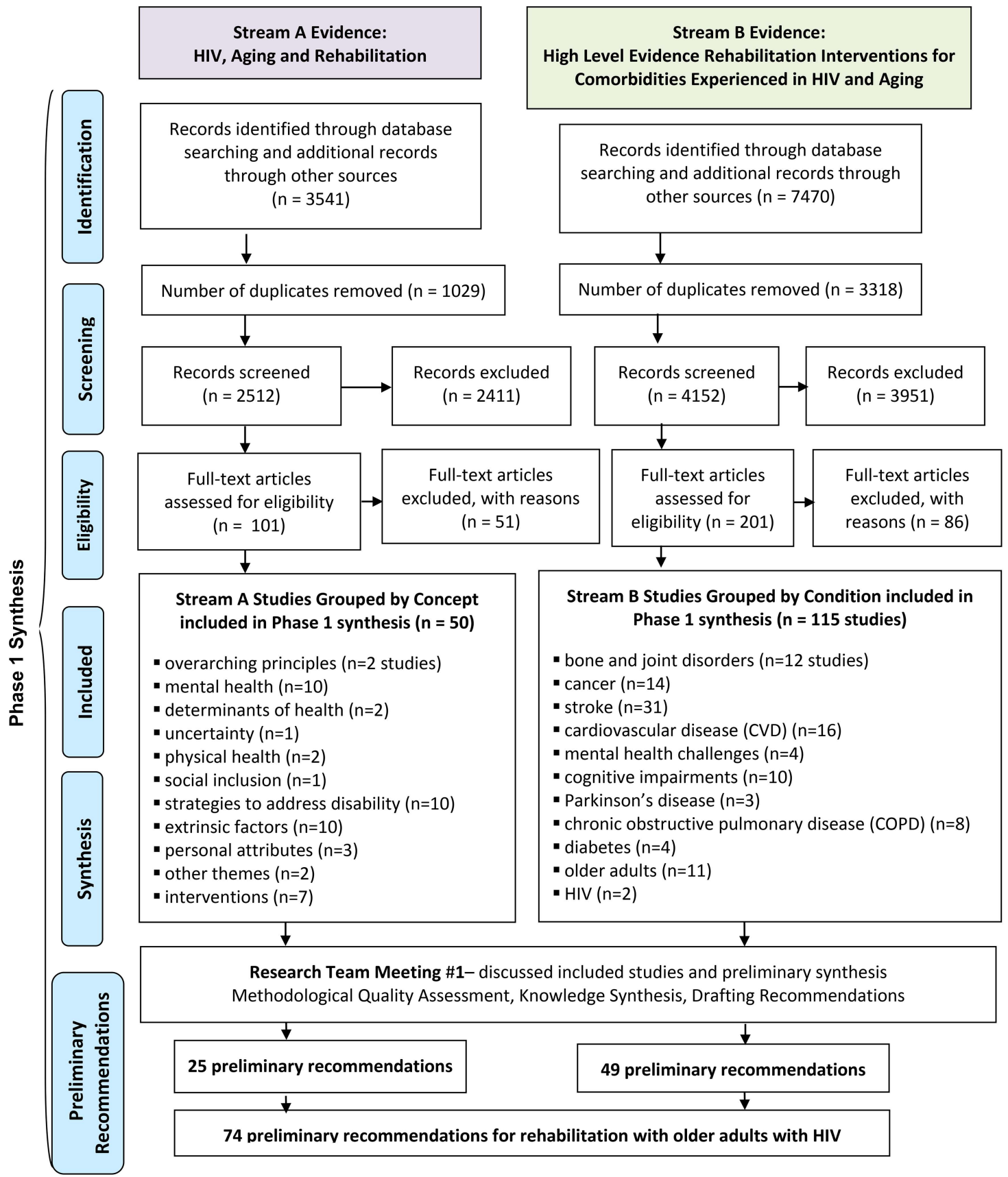

Figure 1 Overview of knowledge synthesis procedure—classification, assessing methodological quality, synthesis and drafting preliminary recommendations (phase 1).

This recommendation would be applicable to some older adults with HIV; Low-minimally endorse or weak recommendation. This recommendation would be applicable to a few older adults with HIV, with potential variability in values and preferences. Wording of this recommendation would include "we suggest, may recommend or may not recommend'; or Very low-do not endorse or do not recommend at all. This recommendation would not be appropriate for older adults living with HIV ( figure 2).

This phase of GRADE rating required a trade-off between benefits and drawbacks, and values and principles of the PLHIV, clinician or researcher. Team members were asked to comment on their values and preferences related to the recommendation and how these influenced their rating. Team members also suggested revisions or refinement to the recommendation.

Collectively the evidence specific to HIV ageing and rehabilitation (stream A) was low to very low quality as much of the evidence consisted of cross-sectional qualitative or quantitative studies (with no comparison group) or narrative reviews. No randomised controlled trials were included. Clinicians and PLHIV on the team incorporated their clinical expertise and experience, values and preferences, respectively, when determining their final GRADE rating. For stream B given only systematic reviews or meta-analyses were included, the 


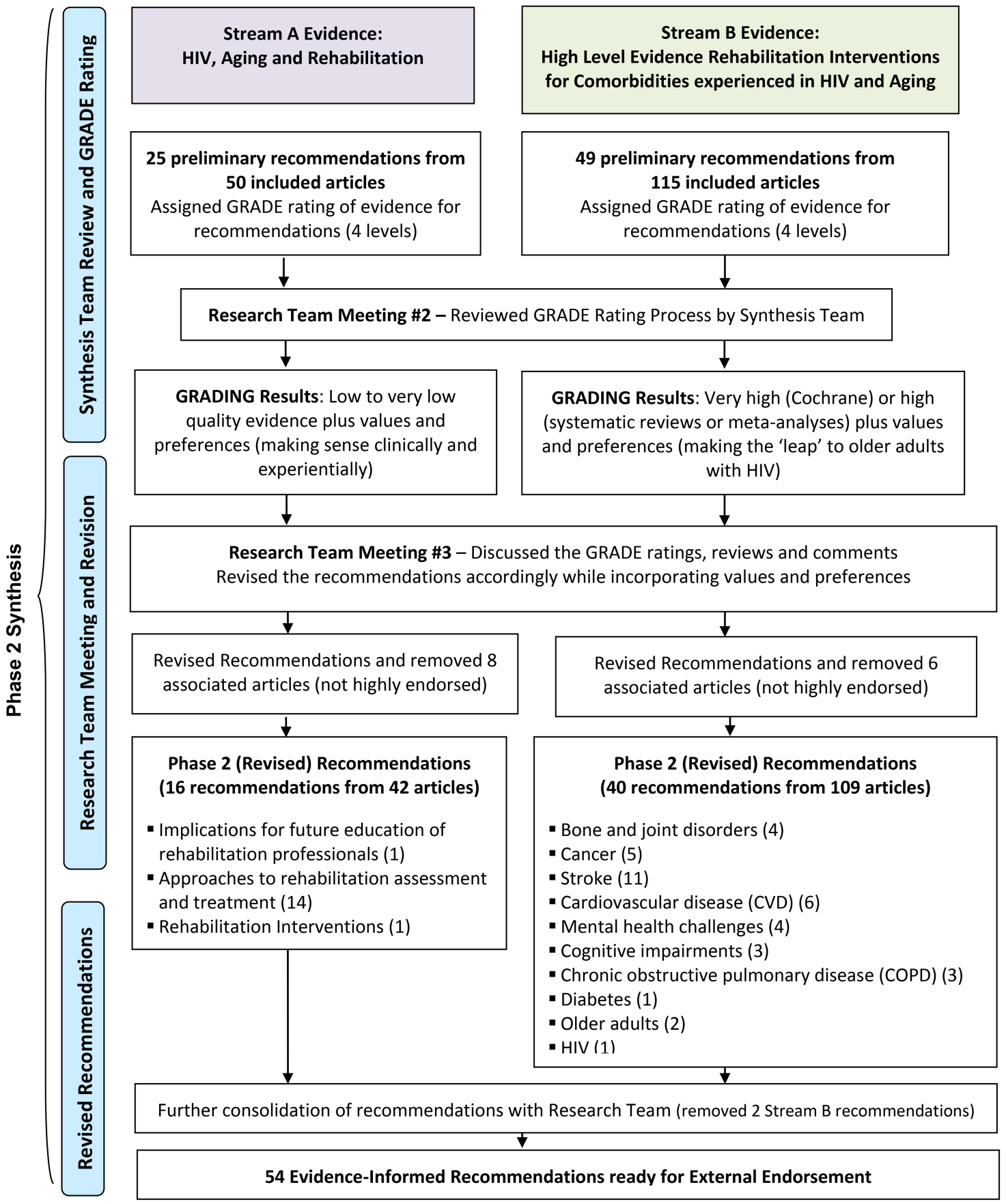

Figure 2 Overview of knowledge synthesis procedure-research team GRADING of recommendations and incorporating values and preferences from PLHIV and clinicians (phase 2).

rating of the evidence was either very high or high. However, the GRADING of the recommendation depended on the extent to which the team felt the evidence was applicable to older adults with HIV and if the intervention posed minimal risk or harm to those living with HIV and these comorbidities.

\section{Phase 2 GRADE results}

The research team met twice to discuss the overall GRADE results and recommendations for revision (Research Team Meetings 2 and 3). In the latter meeting we summarised and incorporated values and preferences of PLHIV and clinicians into the recommendations (figure 2).

\section{Stream A: GRADE rating results and revision}

We consolidated similar or overlapping recommendations and deleted those not highly endorsed by the majority of the team. We also removed recommendations to specific interventions with inconclusive evidence because of team concerns of endorsing specific interventions over others under-reported in the research evidence. 
Overall this process resulted in the deletion of eight articles. The remaining 42 articles in stream A yielded 16 evidence-informed recommendations for older adults with HIV that spanned three themes: (1) implications for future education of rehabilitation professionals (1 recommendation), (2) approaches to rehabilitation assessment and treatment (14 recommendations)and (3) interventions ( 1 recommendation; figure 2 ).

\section{Stream B: GRADE rating results and revision}

On the basis of the GRADE rating of team members and our meeting discussions we revised the stream B recommendations. We deleted recommendations that were not endorsed by the clinicians and PLHIV and recommendations that referred to conditions not common to HIV and ageing. Overall this process resulted in the removal of six articles. The remaining 109 articles in stream B yielded 40 evidence-informed recommendations that spanned the following 10 areas: bone and joint disorders, cancer (general, lung and metastatic cancer), stroke, CVD (myocardial infarction, heart disease, heart failure), mental health challenges, cognitive impairment, COPD, diabetes, older adults and HIV/AIDS (figure 2). Recommendations spanned interventions including exercise, rehabilitation, selfmanagement, cognitive rehabilitation, pulmonary rehabilitation, electrotherapeutic modalities, cardiac rehabilitation, inspiratory muscle training, psychotherapy, models of care and housing models.

\section{Phase 3: External endorsement-incorporating 'expert knowledge' from clinicians and adults living with HIV}

We circulated the recommendations electronically to a broader group of 38 clinicians and PLHIV for external endorsement using an online survey. We asked participants whether they endorsed, did not endorse or had no opinion about each recommendation. Participants were also invited to provide comments. We considered endorsement rates of $>80 \%, 60-80 \%$ and $<60 \%$ as high, moderate and low levels of endorsement, respectively. Responses from this endorsement phase were incorporated into the final revision and refinement of the evidence-informed recommendations (figure 3).

\section{External endorsement results}

Of the 38 PLHIV and clinicians invited to participate in the online endorsement survey, 19 (50\%) completed the online survey. Of the 19 individuals who completed the endorsement survey, $9(47 \%)$ were health professionals, $8(42 \%)$ were PLHIV and $2(11 \%)$ were health professionals and PLHIV. Health professionals included physicians (geriatrics and infectious diseases; 27\%), occupational therapists (27\%), speech-language pathologists $(27 \%)$ and social workers $(18 \%)$.

Rates of endorsement for each recommendation ranged from $47 \%$ (9/19 participants) to $100 \%(19 / 19$ participants). The number of participants who viewed the citations from which the recommendations were derived ranged from 3 (16\%) to 10 participants (53\%). Participants tended to highly endorse recommendations in stream A and those in stream B related to exercise. Recommendations related to inconclusive evidence had lower rates of endorsement. Endorsement participants highlighted how recommendations could be applicable to any population (not just older adults with HIV). Others recommended highlighting other interventions not captured in the recommendations, such as yoga or tai-chi. See online data supplement file 1 for an overview of the endorsement results.

Two recommendations endorsed by $<60 \%$ of participants were removed. The team further synthesised the final 52 specific recommendations into eight overarching recommendations for rehabilitation with older adults living with HIV. See online data supplement file 2 for the final evidence-informed recommendations and online data supplement file 3 for characteristics of included studies in the final recommendations.

\section{Final recommendations}

Results of this synthesis are presented across two streams that represent the two different bodies of research evidence totalling 52 specific recommendations (see online data supplement file 2). We also present overarching recommendations derived from the specific detailed evidence-informed recommendations for rehabilitation with older adults living with HIV (box 1).

\section{Specific (detailed) recommendations}

Stream A results include 16 recommendations derived from 42 research evidence articles specific to rehabilitation with older adults living with HIV. The level of evidence from which these recommendations were derived was either low or very low, meaning the articles were mostly narrative review articles or descriptive studies (either qualitative or quantitative) with small sample sizes. Although the studies were low-level evidence, the PLHIV and clinician endorsements indicated that these were of fundamental importance in management of disability in older adults living with HIV. Stream A recommendations serve as the contextual backdrop to providing rehabilitation care, treatment and support to older adults living with HIV. Some of the recommendations have additional explanatory notes to further explain the context and PLHIV and clinician values (see online data supplement file 2). The recommendations are organised into six categories: (A) preparedness of rehabilitation professionals, (B) approaches to rehabilitation assessment and treatment with older adults living with HIV, (C) extrinsic factors to consider for rehabilitation with older adults living with HIV, (D) intrinsic factors to consider for rehabilitation with older adults living with HIV, (E) rehabilitation approaches and (F) rehabilitation interventions (see online data supplement file 2).

Stream B results include 36 recommendations derived from 108 moderate- or high-level research evidence articles describing the effectiveness of rehabilitation 


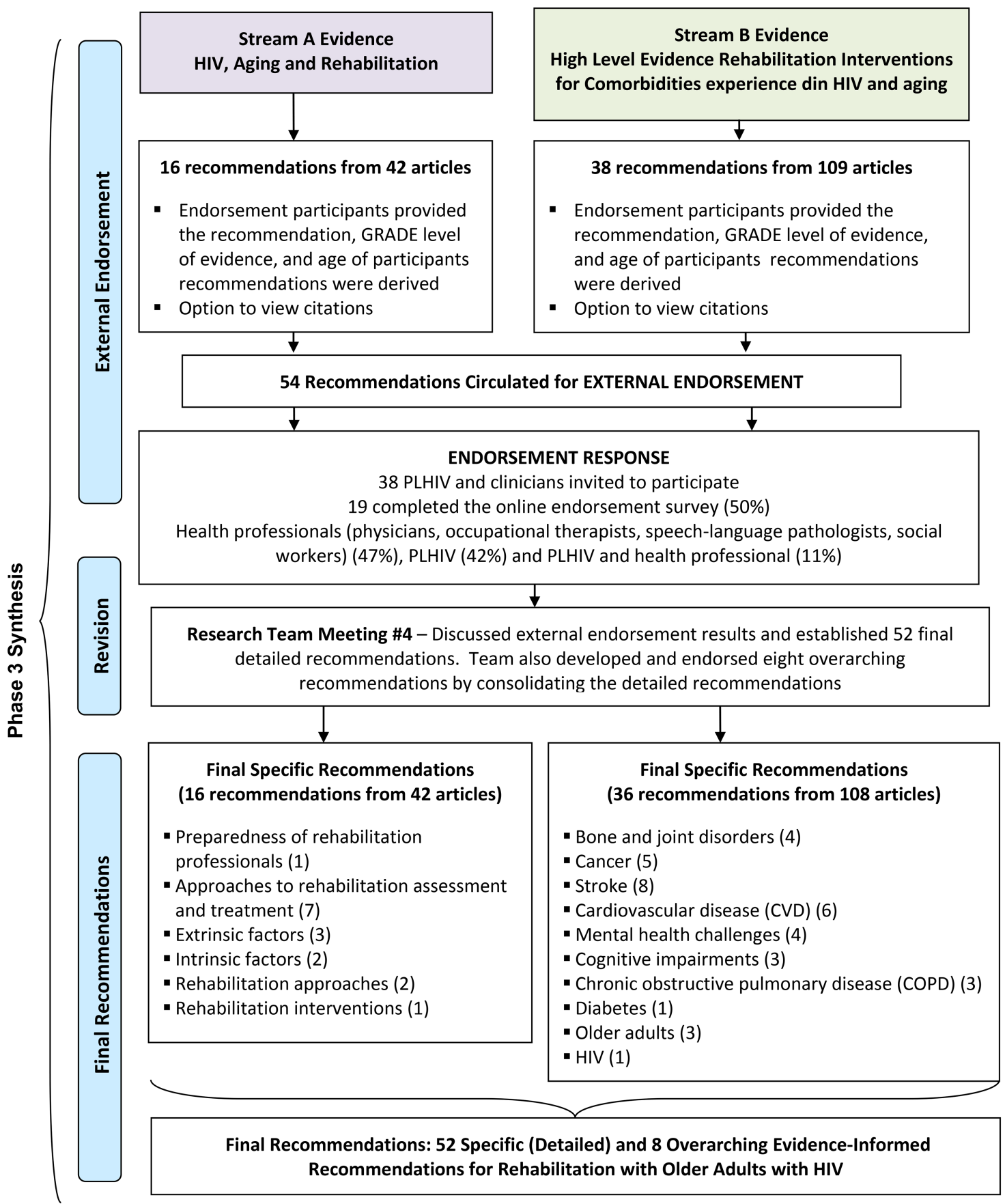

Figure 3 Overview of knowledge synthesis procedure-external endorsement (phase 3).

interventions for adults living with health conditions and include specific considerations when applying rehabilitation interventions for older PLHIV (figure 3). Stream B recommendations include an overview of the prevalence of the condition among older adults with HIV, main health-related challenges for older adults with HIV experiencing this condition from a rehabilitation perspective, study citations, level of evidence from which the recommendation was derived and age of participants included in the evidence (not all high-level rehabilitation intervention evidence was specific to older adults). The recommendations include specific considerations for older adults with HIV. The recommendations are presented based on interventions across (A) older adults, (B) HIV/AIDS and eight common comorbidities that may be experienced by older adults with HIV, (C) bone and joint disorders, (D) cancer, (E) stroke, (F) CVD, (G) mental health challenges, $(\mathrm{H})$ cognitive impairments, (I) COPD and (J) diabetes (see online data supplement file 2).

\section{Overarching recommendations}

To facilitate knowledge transfer and exchange, we established overarching recommendations that summarised 


\section{Box 1 Overarching evidence-informed recommendations for rehabilitation with older adults living with HIV}

The following overarching recommendations provide a general guide to providing rehabilitation care, treatment and support with older adults living with HIV.

Overarching recommendations for rehabilitation with older adults living with HIV

1. Rehabilitation professionals should be prepared to provide care to older adults with HIV who present with complex comorbidities affecting neurological, cardiorespiratory and musculoskeletal systems that may result in physical, mental and social health challenges.

2. Rehabilitation professionals should adopt an individualised and interprofessional approach to practice that is sensitive to the unique values, preferences and needs of older adults with HIV. This approach should include comprehensive assessment and treatment of physical, neurocognitive and mental health impairments, uncertainty (or worrying about the future), functional activity limitations and social exclusion while considering the intersections between personal and social attributes and the broader determinants of health.

3. Multidisciplinary rehabilitation including physical therapy, occupational therapy and speech-language pathology is strongly recommended across the continuum of care (acute, rehabilitation and community-based care) for older adults with HIV to address the multidimensional and episodic nature of disability attributed to HIV and its comorbidities such as bone and joint disorders, cancer, stroke, cardiovascular disease, mental health, cognitive impairment, chronic obstructive pulmonary disease (COPD) and diabetes.

4. Rehabilitation professionals should consider the role of extrinsic contextual factors such as stigma and ageism, HIV disclosure, and emotional and practical social supports on the health and well-being of older adults living with HIV.

5. Rehabilitation professionals should consider the role of intrinsic contextual factors such as self-management and spirituality on the health and well-being of older adults living with HIV.

6. A combination of aerobic and resistive exercise may be recommended for older adults living with HIV who are medically stable and living with comorbidities including bone and joint disorders, cancer, stroke, cardiovascular disease, stroke, mental health, cognitive impairment, COPD and diabetes. The frequency, intensity, time and type of exercise should be individually tailored to the specific goals and capacity of the individual and the specific comorbidity.

7. Cognitive rehabilitation interventions (eg, cognitive training, cognitive stimulation, cognitive rehabilitation) may be recommended for older adults living with HIV with mild cognitive impairment and stroke. Inconclusive or insufficient evidence exists to support the use of cognitive behavioural therapy on older adults with HIV with depression. While cognitive rehabilitation does not appear harmful, weak evidence exists to support the use of cognitive-specific interventions to improve spatial neglect, disability, memory and functional status of older adults who experience stroke. Rehabilitation professionals are encouraged to refer to specific clinical practice guidelines for each health condition to determine the effects of different cognitive interventions on older adults with HIV living with comorbidity.

8. In the absence of high-level evidence on rehabilitation interventions for older adults living with HIV and comorbidities, rehabilitation professionals should refer to existing clinical practice guidelines, systematic reviews, meta-analyses and other forms of high level evidence for recommendations on interventions for a specific comorbidity. These recommendations should be applied using an individualised approach incorporating the unique values, preferences, goals and needs of the individual.

the detailed recommendations in a condensed manner (box 1). We consolidated the 52 specific recommendations into eight overarching recommendations for rehabilitation with older adults living with HIV. These recommendations were endorsed at a final team meeting and provide a broader overview of the evidence synthesis.

\section{DISCUSSION}

We developed evidence-informed recommendations for rehabilitation with older adults living with HIV using a complex knowledge synthesis of two distinct areas of literature while incorporating PLHIV and clinician preferences throughout. To our knowledge, these are the first evidence-informed recommendations for rehabilitation developed specifically for older adults with HIV.

These recommendations may be useful for rehabilitation clinicians who have not worked with PLHIV and HIV specialists unfamiliar with rehabilitation who need an understanding of evidence-informed rehabilitation so that they can make appropriate referrals for their older clients living with HIV. Stream A recommendations were derived from very low-level evidence and result in general statements. Nevertheless, we feel these recommendations are useful in addressing an overall approach to working with older adults with HIV. The low level of evidence derived from this area of literature highlights the paucity of evidence specific to rehabilitation of older adults with HIV and indicates the need for increased work in this area. While our focus was on older adults with HIV, many of the stream B recommendations were derived from evidence not specific to older adults. The wording of our recommendations depended on how well, or to what extent we could make the 'leap' from the condition-specific evidence to a recommendation for rehabilitation specific to older adults living with HIV and its comorbidities. We included an overview of the prevalence of comorbidities among older adults with HIV to assist clinicians in implementing the recommendations among adults with HIV living with comorbidities. $^{30}$ The supportive notes that augment the recommendations were derived primarily from PLHIV and clinician values and preferences to help situate the recommendation into the context of older adults with HIV. Rehabilitation professionals often tailor treatment strategies to address the consequences of disease (disability) using an individualised goal-setting approach that considers the unique health and social challenges experienced by older adults with HIV. In the absence of 
high-level evidence on rehabilitation interventions specific to older adults with HIV, clinicians may refer to the existing guidelines for a specific comorbidity, and incorporate an individualised approach to assessment and treatment.

We chose to present a combination of specific and overarching recommendations to guide rehabilitation with older adults with HIV. Those working with older adults with a specific comorbidity may find the detailed recommendations useful to their practice. Although specific recommendations are more likely to be followed, ${ }^{31}$ we feel the consolidated (overarching) recommendations may be useful to health providers less familiar working in HIV care and well suited for knowledge translation to a broader health provider audience and community-based organisations.

Overall strengths of our approach included our unique synthesis of two distinct areas of literature combining lower level evidence on emerging issues of HIV and ageing with higher level evidence on comorbidities commonly experienced by PLHIV to provide a strong foundation for the development of evidence-informed recommendations. We used a systematic approach in identifying literature, determining inclusion, data extraction, and drafting and refining the recommendations. We drafted the recommendations to include clear actionable and precise terminology, associated with the level of evidence available. We included specific citations from which the recommendation was derived so readers may refer to the original evidence source of the recommendation. ${ }^{32}$

Our interprofessional and community-integrated approach involving 'expert' older PLHIV and clinicians brought a diverse group of stakeholders together on numerous occasions to engage in the iterative process of recommendation development, review and refinement and ensured the recommendations were practical and relevant to the HIV community. External endorsement further integrated PLHIV and clinician preferences into assessing the feasibility and refinement of recommendations for use in HIV practice. ${ }^{30}$ Knowledge, values and experiences of clinicians and PLHIV were integral in the development of the recommendations, particularly when determining the relevance or unique considerations when devising recommendations from evidence derived from other chronic conditions. Our community-engaged approach involved PLHIV as members of the core research team, as well as participants in the external endorsement phase. This form of community-academicclinical research partnership is growing in prominence because it strengthens the potential for effective knowledge transfer and exchange in health research. ${ }^{33}$

Challenges of this synthesis included combining two areas of research evidence that differed in quality and context. We chose to retain two parallel but distinct syntheses presented as one collective set of recommendations enabling us to synthesise emerging lower level evidence on HIV ageing and rehabilitation with higher level more established evidence in chronic diseases experienced by older adults with HIV. ${ }^{34}$ Much of the evidence from which these recommendations were derived is from the United States, hence the generalisability of these recommendations to other contexts is unknown. The lack of high-level stream A evidence specific to HIV, ageing and rehabilitation resulted in high-level considerations when working with older adults with HIV, and emphasises the need for further rehabilitation intervention research specific to older adults with HIV Disparities emerged between evidence considered weak by GRADE definition, but essential to the values and preferences of PLHIV and clinicians. We were uncertain how to weight the research evidence with PLHIV and clinician values and preferences in order to establish the strength of a given recommendation. We chose to remove recommendations for rehabilitation approaches with weak evidence that were not highly endorsed by the majority of team members. Finally, these evidence-informed recommendations do not specifically address the issue of caregiving, respite and potential caregiver burnout, important issues that should be considered by clinicians in the context of HIV and ageing. ${ }^{35}$

The development of these recommendations is timely given the changing demographic of adults ageing with HIV. These recommendations directly address key research priorities on comorbidities and access to rehabilitation identified in a national scoping study of the Canadian Working Group on HIV and Rehabilitation (CWGHR). ${ }^{36}$ Our recommendations also address key issues related to HIV, rehabilitation and ageing that emerged from a national consultation with PLHIV, researchers, educators, clinicians and policy stakeholders by CWGHR including comorbidities experienced by older PLHIV and social determinants of health. ${ }^{37}$ These issues similarly emerged from the external endorsement whereby participants also indicated the importance of end-of-life care, ${ }^{38}$ lifestyle modifications including adoption of exercise and yoga ${ }^{39} 40$ and smoking cessation among older adults with $\mathrm{HIV}^{41}$ as critical for consideration in the care and prevention strategies to enhance the health of older PLHIV. Moreover, while evidence describes potential benefits of supplements used in osteoarthritis ${ }^{42}$ or central nervous stimulants to alleviate HIV-associated cognitive impairments and fatigue, ${ }^{43} 44$ the focus of these rehabilitation recommendations were non-pharmacological in nature. We developed these recommendations in accordance with the principles outlined by CWGHR for the development of guidelines for rehabilitation in the context of HIV. ${ }^{45}$ Merging the traditionally separate areas of rehabilitation, HIV and disability enabled us to create evidence-informed recommendations that are relevant for rehabilitation in the context of HIV and provide clear actionable recommendations that could direct future practice. ${ }^{45}$

Limitations of this research included the qualitative nature of the synthesis whereby we were unable to pool results from included studies into meta-analyses. We may 
have missed other high-level evidence on rehabilitation interventions such as fall prevention or balance training that were not specific to our predetermined comorbidities but may be experienced by older adults living with multiple comorbidities. Rehabilitation interventions clinicians use in practice which are beneficial to older adults with HIV may not have been captured in this synthesis due to the paucity of HIV and ageing literature (stream A) or due to the lack of high level of evidence (stream B). Finally, HIV-specific evidence on rehabilitation of older adults with HIV continues to emerge since we conducted our literature search for included studies in 2011. Recent evidence suggests cognitive rehabilitation interventions such as computerised speed of processing training and selfgeneration strategies can enhance verbal recall and cognitive function among older adults with HIV, and that interventions to promote self-efficacy and social support may enhance health-related quality of life among older men with HIV. ${ }^{46-49}$ Ongoing revision of the recommendations will be required to reflect the emerging evidence and changing needs of older adults living with HIV.

\section{CONCLUSIONS}

We established 8 overarching and 52 specific evidence-informed recommendations from a combination of low-level evidence specific to HIV, ageing and rehabilitation and high-level research evidence describing the effectiveness of rehabilitation interventions for comorbidities experienced by older adults with HIV. PLHIV and clinician values and preferences were integral in developing these recommendations. These recommendations address approaches to rehabilitation assessment and interventions, and contextual factors to consider for rehabilitation with older adults living with HIV. These evidence-informed recommendations provide a guide for rehabilitation with older adults living with HIV.

\section{Author affiliations}

${ }^{1}$ Department of Physical Therapy, University of Toronto, Toronto, Ontario, Canada

${ }^{2}$ School of Rehabilitation Science, McMaster University, Hamilton, Ontario, Canada

${ }^{3}$ Canadian Working Group on HIV and Rehabilitation, Toronto, Ontario,

\section{Canada}

${ }^{4}$ Department of Occupational Science and Occupational Therapy, University of Toronto, Toronto, Ontario, Canada

${ }^{5}$ AIDS Committee of Toronto, Toronto, Ontario, Canada

${ }^{6}$ St Michael's Hospital, Toronto, Ontario, Canada

${ }^{7}$ Faculty of Medicine, University of Manitoba, Winnipeg, Manitoba, Canada

${ }^{8}$ National Hospital for Neurology and Neurosurgery, University College

Hospitals NHS Foundation Trust, London, UK

${ }^{9}$ Women's College Hospital, Toronto, Ontario, Canada

Acknowledgements The authors gratefully acknowledge Elizabeth Uleryk (Hospital for Sick Children) for her role in the search strategy; Ken King (Canadian Working Group on HIV and Rehabilitation) and Sheila Thomas (St Michael's Hospital) for their role as knowledge users in earlier phases of this work; Jenn Jimmo for her role in data extraction; Nicole Gervais for her involvement in the implementation of the endorsement survey and Nkem Iku for her involvement in the literature review, endorsement survey summary and the formatting of the recommendations. The authors also thank David Vance and Debra Henninger, authors of included evidence who graciously provided additional information for this synthesis. The authors acknowledge the following individuals for their involvement in the endorsement phase of the recommendations development: Colleen Price, Glyn Townson (Canadian Working Group on HIV and Rehabilitation), Rob Alexander (HIVER Consulting), Charles Furlotte (Hamilton Health Sciences Centre, McMaster University), Dawn James (Nine Circles Community Health Centre), Francisco Ibáñez-Carrasco (Ontario HIV Treatment Network), Gordon Arbess (St Michael's Hospital), Bani Ahuja (Occupational Therapist), Michael Hamilton, Evan Collins (Department of Psychiatry, University of Toronto), and Murray Jose (Toronto People With AIDS Foundation).

Contributors KKOB and PS led the conceptual design of the study, acquisition of funding, conducted the synthesis and drafted the manuscript. KKOB, PS, AMT, DM and BT reviewed evidence for inclusion; KKOB, PS, BT, AMT and DM extracted data from included studies; KKOB, AMT, PS and BT conducted the initial methodological quality assessment and primary synthesis; LB, BT, DM, AC, WC, GR, JW and TT were involved in the review and GRADING of the recommendations, analytical interpretations, endorsement and refinement of the recommendations. JM provided overall guidance on the synthesis methodology. EZ was the principal knowledge user and advised on the overall development and process for future translation of the recommendations. All authors read and approved the final manuscript.

Funding This research was funded by a Canadian Institutes of Health Research (CIHR) Knowledge Synthesis grant (Fund \# 108046). At the time of this study, KKOB was funded by a fellowship from the CIHR and a Michael DeGroote Postdoctoral Fellowship (McMaster University). KKOB is funded by a CIHR New Investigator Award, HIV/AIDS Research Initiative.

\section{Competing interests None.}

Ethics approval This research received Research Ethics Board approval from McMaster University, Hamilton, Ontario, Canada.

Provenance and peer review Not commissioned; externally peer reviewed.

Data sharing statement No additional data are available.

Open Access This is an Open Access article distributed in accordance with the Creative Commons Attribution Non Commercial (CC BY-NC 3.0) license, which permits others to distribute, remix, adapt, build upon this work noncommercially, and license their derivative works on different terms, provided the original work is properly cited and the use is non-commercial. See: http:// creativecommons.org/licenses/by-nc/3.0/

\section{REFERENCES}

1. Kirk JB, Goetz MB. Human immunodeficiency virus in an aging population, a complication of success. J Am Geriatr Soc 2009;57:2129-38.

2. Oursler KK, Goulet JL, Leaf DA, et al. Association of comorbidity with physical disability in older HIV-infected adults. AIDS Patient Care STDS 2006:20:782-91.

3. Weiss JJ, Osorio G, Ryan E, et al. Prevalence and patient awareness of medical comorbidities in an urban AIDS clinic. AIDS Patient Care STDS 2010;24:39-48.

4. Willard S, Holzemer WL, Wantland DJ, et al. Does 'asymptomatic' mean without symptoms for those living with HIV infection? AIDS Care 2009;21:322-8.

5. O'Brien KK, Bayoumi AM, Strike C, et al. Exploring disability from the perspective of adults living with HIV/AIDS: development of a conceptual framework. Health Qual Life Outcomes 2008;6:76.

6. Rusch M, Nixon S, Schilder A, et al. Impairments, activity limitations and participation restrictions: prevalence and associations among persons living with HIV/AIDS in British Columbia. Health Qual Life Outcomes 2004;2:46.

7. Rusch M, Nixon S, Schilder A, et al. Prevalence of activity limitation among persons living with HIV/AIDS in British Columbia. Can J Public Health 2004;95:437-40.

8. Vance DE, Mugavero M, Willig J, et al. Aging with HIV: a cross-sectional study of comorbidity prevalence and clinical characteristics across decades of life. J Assoc Nurses AIDS Care 2011;22:17-25. 
9. Brown TT, McComsey GA. Osteopenia and osteoporosis in patients with HIV: a review of current concepts. Curr Infect Dis Rep 2006;8:162-70.

10. Heaton RK, Clifford DB, Franklin DR Jr, et al. HIV-associated neurocognitive disorders persist in the era of potent antiretroviral therapy: CHARTER study. Neurology 2011;75:2087-96.

11. Shiels MS, Cole SR, Kirk GD, et al. A meta-analysis of the incidence of non-AIDS cancers in HIV-infected individuals. J Acquir Immune Defic Syndr 2009;52:611-22.

12. Heckman TG, Heckman BD, Kochman A, et al. Psychological symptoms among persons 50 years of age and older living with HIV disease. Aging Mental Health 2002;6:121-8.

13. Grov C, Golub SA, Parsons JT, et al. Loneliness and HIV-related stigma explain depression among older HIV-positive adults. AIDS Care 2010;22:630-9.

14. Vance DE, Woodley RA. Strengths and distress in adults who are aging with HIV: a pilot study. Psychol Rep 2005;96:383-6.

15. Oursler KK, Sorkin JD, Smith BA, et al. Reduced aerobic capacity and physical functioning in older HIV-infected men. AIDS Res Hum Retroviruses 2006;22:1113-21.

16. Oursler KK, Goulet JL, Crystal S, et al. Association of age and comorbidity with physical function in HIV-infected and uninfected patients: results from the Veterans Aging Cohort Study. AIDS Patient Care STDS 2011;25:13-20.

17. Worthington $\mathrm{C}$, Myers T, O'Brien $\mathrm{K}$, et al. Rehabilitation in HIV/AIDS development of an expanded conceptual framework. AIDS Patient Care STDS 2005;19:258-71.

18. Jolliffe JA, Rees K, Taylor RS, et al. Exercise-based rehabilitation for coronary heart disease. Cochrane Database Syst Rev 2001;1: CD001800.

19. Saunders DH, Greig CA, Mead GE, et al. Physical fitness training for stroke patients. Cochrane Database Syst Rev 2009;4:CD003316.

20. Cramp F, Daniel J. Exercise for the management of cancer-related fatigue in adults. Cochrane Database Syst Rev 2008;2:CD006145.

21. Worthington C, Myers T, O'Brien K, et al. Rehabilitation professionals and human immunodeficiency virus care: results of a national Canadian survey. Arch Phys Med Rehabil 2008;89:105-13.

22. van der Weijden T, Legare F, Boivin A, et al. How to integrate individual patient values and preferences in clinical practice guidelines? A research protocol. Implement Sci 2010;5:10.

23. Higgins J, Green S. Cochrane handbook for systematic reviews of interventions. Cochrane Collaboration. Version 5.0.2 [updated September 2009]. 2009.

24. Atkins D, Best D, Briss PA, et al. Grading quality of evidence and strength of recommendations. BMJ 2004;328:1490.

25. Guyatt $\mathrm{G}$, Gutterman D, Baumann $\mathrm{MH}$, et al. Grading strength of recommendations and quality of evidence in clinical guidelines: report from an American College of Chest Physicians Task Force. Chest 2006;129:174-81.

26. Brozek JL, Akl EA, Alonso-Coello P, et al. Grading quality of evidence and strength of recommendations in clinical practice guidelines. Part 1 of 3 . An overview of the GRADE approach and grading quality of evidence about interventions. Allergy 2009;64:669-77.

27. Brozek JL, Akl EA, Jaeschke R, et al. Grading quality of evidence and strength of recommendations in clinical practice guidelines: Part 2 of 3 . The GRADE approach to grading quality of evidence about diagnostic tests and strategies. Allergy 2009;64:1109-16.

28. Brozek JL, Akl EA, Compalati E, et al. Grading quality of evidence and strength of recommendations in clinical practice guidelines part 3 of 3 . The GRADE approach to developing recommendations. Allergy 2011;66:588-95.

29. Hsieh HF, Shannon SE. Three approaches to qualitative content analysis. Qual Health Res 2005;15:1277-88.
30. Shekelle P, Woolf S, Grimshaw JM, et al. Developing clinical practice guidelines: reviewing, reporting, and publishing guidelines; updating guidelines; and the emerging issues of enhancing guideline implementability and accounting for comorbid conditions in guideline development. Implement Sci 2012;7:62.

31. Grol R, Dalhuijsen J, Thomas S, et al. Attributes of clinical guidelines that influence use of guidelines in general practice: observational study. BMJ 1998;317:858-61.

32. Woolf S, Schunemann HJ, Eccles MP, et al. Developing clinical practice guidelines: types of evidence and outcomes; values and economics, synthesis, grading, and presentation and deriving recommendations. Implement Sci 2012;7:61.

33. Rhodes SD, Malow RM, Jolly C. Community-based participatory research: a new and not-so-new approach to HIV/AIDS prevention, care, and treatment. AIDS Educ Prev 2010;22:173-83.

34. Turner-Stokes L. Evidence for the effectiveness of multi-disciplinary rehabilitation following acquired brain injury: a synthesis of two systematic approaches. J Rehabil Med 2008;40:691-701.

35. Emlet CA, Poindexter CC. Unserved, unseen, and unheard: integrating programs for HIV-infected and HIV-affected older adults. Health Soc Work 2004;29:86-96.

36. O'Brien K, Wilkins A, Zack E, et al. Scoping the field: identifying key research priorities in HIV and rehabilitation. AIDS Behav 2010;14:448-58.

37. Canadian Working Group on HIV and Rehabilitation. Partners in aging: report on a national project on HIV and aging. 2010.

38. Harding $R$, Karus D, Easterbrook $P$, et al. Does palliative care improve outcomes for patients with HIV/AIDS? A systematic review of the evidence. Sex Transm Infect 2005;81:5-14

39. Yahiaoui A, McGough EL, Voss JG. Development of evidence-based exercise recommendations for older HIV-infected patients. J Assoc Nurses AIDS Care 2012;23:204-19.

40. Cade WT, Reeds DN, Mondy KE, et al. Yoga lifestyle intervention reduces blood pressure in HIV-infected adults with cardiovascular disease risk factors. HIV Med 2010;11:379-88.

41. Nahvi S, Cooperman NA. Review: the need for smoking cessation among HIV-positive smokers. AIDS Educ Prev 2009;21:14-27.

42. Salazar J, Bello L, Chavez M, et al. Glucosamine for osteoarthritis: biological effects, clinical efficacy, and safety on glucose metabolism. Arthritis 2014;2014:432463.

43. Breitbart W, Rosenfeld B, Kaim M, et al. A randomized, double-blind placebo-controlled trial of psychostimulants for the treatment of fatigue in ambulatory patients with human immunodeficiency virus disease. Arch Intern Med 2001;161:411-20.

44. Hinkin $\mathrm{CH}$, Castellon SA, Hardy DJ, et al. Methylphenidate improves HIV-1-associated cognitive slowing. J Neuropsychiatry Clin Neurosci 2001;13:248-54.

45. O'Brien KK, Wilkins A, Zack E, et al. Developing clinical practice guidelines in HIV rehabilitation: process recommendations and guiding principles. AIDS Educ Prev 2011;23:457-68.

46. Vance DE, Fazeli PL, Ross LA, et al. Speed of processing training with middle-age and older adults with HIV: a pilot study. $J$ Assoc Nurses AIDS Care 2012;23:500-10.

47. Weber E, Woods SP, Kellogg E, et al. Self-generation enhances verbal recall in individuals infected with HIV. J Int Neuropsychol Soc 2012;18:128-33.

48. Weber E, Blackstone K, Woods SP. Cognitive neurorehabilitation of HIV-associated neurocognitive disorders: a qualitative review and call to action. Neuropsychol Rev 2013;23:81-98.

49. Emlet CA, Fredriksen-Goldsen KI, Kim HJ. Risk and protective factors associated with health-related quality of life among older gay and bisexual men living with HIV disease. Gerontologist 2013;53:963-72. 